\title{
(6) OPEN ACCESS \\ How do HIV-negative individuals in sub-Saharan Africa change their sexual risk behaviour upon learning their serostatus? A systematic review
}

\author{
Sanjeev Ramachandran, ${ }^{1}$ Sharmistha Mishra, ${ }^{1,2}$ Natalie Condie, ${ }^{1}$ Michael Pickles ${ }^{1}$
}

\begin{abstract}
- Additional material is published online only. To view please visit the journal online (http://dx.doi.org/10.1136/ sextrans-2015-052354).

${ }^{1}$ Department of Infectious Disease Epidemiology, Imperial College London, London, UK

${ }^{2}$ Division of Infectious Diseases, Department of Medicine, St Michael's Hospital, University of Toronto, Toronto, Canada
\end{abstract}

\section{Correspondence to}

Dr Michael Pickles, Department of Infectious Disease

Epidemiology, St Mary's

Campus, Norfolk Place,

Imperial College, London W2

1PG, UK;

m.pickles@imperial.ac.uk

Received 26 August 2015

Revised 12 May 2016

Accepted 22 May 2016

Published Online First

17 August 2016

\section{Linked}

- http://dx.doi.org/10.1136/ sextrans-2016-052640

- http://dx.doi.org/10.1136/ sextrans-2016-052651

\section{CrossMark}

To cite: Ramachandran $S$, Mishra S, Condie N, et al. Sex Transm Infect 2016:92:571-578
ABSTRACT

Objective To determine whether, and how, sexual behaviour of HIV-negative individuals in sub-Saharan Africa (SSA) changes upon learning their serostatus. Methods We systematically reviewed the published literature using EMBASE and Medline to search for publications between 2004 and 2014. We included studies that quantified behaviour change (condom use, number of sexual partners or sex acts) following an HIV test in HIV-negative adults in SSA, and extracted relevant data including study characteristics and measurement type.

Results From 2185 unique citations, $\mathrm{n}=14$ studies representing 22390 participants met our inclusion criteria. We did not pool data due to marked heterogeneity in study outcome measures. The proportion of participants reporting consistent condom use $(n=6)$ post-testing ranged from $7.6 \%$ greater, to $10.6 \%$ fewer, while 'no condom use' $(n=5)$ ranged from $40.0 \%$ less, to $0.7 \%$ more. Condom use in serodiscordant couples increased $(n=3)$. Five studies measured the proportion reporting abstinence, finding an increase of $10.9 \%$ to a decrease of $5.3 \%$ post-testing. The post-testing change in the mean number of sex acts $(n=3)$ ranged from a relative decrease of $15.7 \%$ to a relative increase of $9.4 \%$. Two studies reported relative decreases in the mean number of sexual partners of $35.2 \%$ and $14.0 \%$. Three studies examining serodiscordant primary relationships specifically all showed increases in extrarelational sex.

Conclusions With the exception of serodiscordant couples, there is variable evidence that awareness of one's serostatus leads to substantial changes in risk behaviour among HIV-negative individuals. Further research is needed to estimate the behavioural impact of learning one's serostatus in SSA.

\section{INTRODUCTION}

Behavioural interventions remain important for HIV prevention in sub-Saharan Africa (SSA)-a region accounting for $70 \%$ of HIV cases worldwide. ${ }^{1}$ HIV testing offers an opportunity to promote safer sexual behaviour through counselling. The potential for antiretroviral therapy (ART) to reduce transmission ${ }^{2}$ has led to intensifying of HIV testing efforts, ${ }^{3}$ with a focus on positive prevention $^{4}$ where HIV-positive individuals are targeted for prevention efforts. However, it remains important to understand behavioural changes after individuals receive an HIV-negative test result.

Mathematical models suggest that universal testing and treatment, where all are offered HIV testing and counselling with immediate ART for those with HIV, could substantially reduce HIV incidence. ${ }^{5-9}$ However, these models rarely include potential behaviour changes of HIV-negative individuals posttesting, ${ }^{5-7}$ and when they do the parameter inputs are either assumed, ${ }^{9}$ or are based on a few studies conducted before $2000 .^{8}$ As more people become aware of their serostatus, it is important to understand how the awareness and associated counselling impact the larger HIV-negative population.

Three systematic reviews have previously examined the impact of voluntary counselling and testing (VCT) on the sexual behaviours of clinic attendees in low-income and middle-income countries. ${ }^{10-12}$ Two estimated outcomes by serostatus, finding that the effects (change in condom use; number of sexual partners) were not statistically significant when restricted to HIV-negative persons. $^{11}{ }^{12}$ When considering HIV-positive and HIV-negative individuals jointly, all three studies found that VCT positively influences risk behaviour, including increased condom use $\mathrm{s}^{10-12}$ and reduced number of sexual partners. ${ }^{11}{ }^{12}$ The reviews were all restricted to data collected between 1990 and $2005-2006^{10} 11$ or $2010 .^{12}$ To our knowledge, there have been no systematic reviews focused on HIV-negative populations in SSA in the era following the scale-up of HIV testing and ART, which began in $2003 .{ }^{13}$ Changes in HIV test delivery (such as opt-out systems), improved on-treatment survival among HIVpositive individuals, and increases in HIV awareness and knowledge all occurred around this time, and may have influenced how individuals perceived HIV severity and responded to HIV-negative test results.

We sought to determine how the sexual behaviour of HIV-negative individuals in SSA changes after learning their serostatus in this period after ART roll-out (post-2003).

\section{METHODS}

\section{Study selection}

We systematically reviewed the peer-reviewed, published literature according to the Preferred Reporting Items for Systematic Reviews and Meta-Analyses guidelines. ${ }^{14}$ We searched two electronic databases using the search terms detailed in the online supplementary material: EMBASE and Medline for articles published between 1 January 2004 and 8 November 2014, with no language restrictions. Reference lists of included studies were not screened to identify further data.

After screening titles and abstracts, we performed a full-text review of remaining articles. The 
screening and full-text review were conducted by two independent reviewers (SR and NC), with arbitration in case of disagreement (MP). We contacted authors for clarifications when consensus could not be reached after full-text review, or when appropriate data on pre-test and post-test behaviour was present but not stratified by serostatus.

The inclusion criteria were as follows: (1) studies conducted in SSA as defined by the United Nations Statistics Division; ${ }^{15}$ (2) participants enrolled after 1 January 2003; (3) serostatus defined by documented HIV test result (not self-report); (4) participants $\geq 15$ years of age; (5) quantitative measurement on at least one behavioural outcome collected before and after HIV testing. Thus, inclusion criteria were restricted to 'pre/post', serial cross-sectional, prospective cohort and time series studies. During screening and full-text review, a study was excluded once it met any exclusion criterion. The exclusion criteria were always applied in the order given in the online supplementary material. Studies focused exclusively on persons who inject drugs were excluded.

\section{Types of outcome}

Outcomes were divided into three domains: condom use, number of sexual partners and number of sex acts (see online supplementary material for more detail). In the later two domains, zero partners or zero sex acts were defined as abstinence.

We give a descriptive analysis of the outcomes, and report the absolute change in outcomes between baseline and follow-up unless otherwise stated. In studies reporting outcomes over multiple follow-up periods, we report the latest recorded outcome to represent longer-term changes. Data over other periods are reported in the online supplementary material. The number of studies is indicated by n. We did not pool outcomes due to the marked heterogeneity in study outcome measures, particularly in the follow-up period and study populations.

We evaluated four types of potential bias in the primary studies: sampling bias, social desirability bias, recall bias and attrition bias. For each study, we assigned a qualitative assessment of whether it was at risk of bias for the outcome of interest, based on literature. ${ }^{16-18}$ Details are provided in the online supplementary material.

\section{RESULTS}

Of 2185 unique records, we excluded 1960 by abstract/title screening, and 210 by full-text review (see online supplementary figure S1). Fourteen studies met inclusion criteria, and provided outcomes on $22390 \mathrm{HIV}$-negative adults in SSA.

\section{Description of studies}

Table 1 describes the characteristics of included studies. Settings included southern and eastern Africa, primarily Uganda, ${ }^{19-26}$ South Africa ${ }^{23} 27-30$ and Tanzania. ${ }^{23} 28{ }^{30}$ Five studies covered multiple countries. $^{23} 25{ }^{28-30}$ The recruitment setting was community-based $\quad(n=5),{ }^{19} \quad 22 \quad 24 \quad 28 \quad 30 \quad$ clinic-based $(\mathrm{n}=3),{ }^{20} 2127$ a combination of community-based and clinicbased $(n=5)^{23} 25262931$ and workplace-based in one study. ${ }^{32}$ The majority of studies were prospective cohorts $(\mathrm{n}=9),{ }^{20} 21232426-283132$ while the remainder were randomised controlled trials $(\mathrm{n}=5) .{ }^{19} 22252930$

In seven studies, the primary purpose was to evaluate changes in sexual behaviour following HIV testing. ${ }^{20} \quad 23-25 \quad 27 \quad 31 \quad 32$ Participants in all 14 studies underwent either individual $^{19} 20222426-32$ or couples ${ }^{21}$ counselling, or a combination of both. $^{23} 25$ Of studies specifying whether counselling was pre- testing or post-testing, two used post-test counselling only, ${ }^{20} 32$ while two used both. ${ }^{25} 29$

\section{Description of participants}

Six studies recruited sexually active adults from the general population $^{19} 2^{22} 2^{28-31}$ (one recruited half the sample from bar and hotel workers, regarded as higher risk individuals ${ }^{28}$ ), four evaluated HIV-negative partners in serodiscordant relationships, ${ }^{21} 232526$ one focused on STI clinic attendees, ${ }^{27}$ one on general outpatients, ${ }^{20}$ one on employees ${ }^{32}$ and one on nonspousal household members of those initiating ART. ${ }^{24}$ No study specifically enrolled key populations, aside from seronegative partners in serodiscordant partnerships, or reported data by risk factors such as formal sex work. The minimum age of recruitment ranged from $15^{19} 22$ to 18 years. Five studies did not set a maximum age limit, ${ }^{20} 23272832$ while in those that did it varied from $35^{31}$ to $69^{24}$ years.

One largely unexplored issue is participants' prior testing history. Only three studies in this review reported how many participants had received prior VCT. ${ }^{19} 2022$

\section{Risk of potential bias}

The majority of studies used face-to-face interviews, so were judged to be at risk of social desirability bias $(n=12)$. The rest used audio-computer-assisted structured interview ${ }^{27}$ and computer-assisted personal interview ${ }^{20}$ to ascertain self-reported behavioural data, reflecting reduced risk of social desirability bias. All studies were at risk of sampling bias, due to the use of convenience sampling or recruitment from specific locations such as clinics. Nine studies were at risk of recall bias, ${ }^{19-22} 2426293132$ while only one study ${ }^{24}$ was deemed at risk of attrition bias. A full list of the potential biases of each study is given in table 1. Six studies were at higher risk of confounding as the primary study outcome was not to measure changes in behaviour post-testing. All studies were at risk of temporal confounding, owing to the pre-post design used.

\section{HIV testing and condom use}

Thirteen studies presented data on condom use (table 2; additional time-points in online supplementary table S1).

Six studies measured consistent condom use, reporting one of 'always $100 \%$ using a condom', 202128 'consistent condom use'19 22 and 'no unprotected sex'. ${ }^{32}$ Baseline levels of consistent condom use were low (eg, $0.3 \%-33.7 \%$ reported consistent condom use in the last year, $n=3$ ). The post-testing change in the proportion of participants reporting consistent condom use ranged from $7.6 \%$ greater, $^{20}$ to $10.6 \%$ fewer $^{21}$ (median across studies: decrease of $0.2 \% ; 2 / 6$ studies showed an increase).

Between baseline and follow-up, the change in the proportion of participants reporting 'no condom use' ranged from $40.0 \%$ less $^{21}$ to $0.7 \%$ more $^{28}$ (median: decrease by $2.2 \%, 4 / 5$ studies showed a decrease). Two studies found decreases of $19.8 \%{ }^{20}$ and $16.6 \%,{ }^{24}$ respectively in the proportion of participants engaging in condomless sex with an HIV-positive partner or a partner whose HIV status was unknown. In the proportion of participants not using a condom at last sex increased by $8 \%$ in the intervention arm, but decreased by $15 \%$ in the control $\operatorname{arm}^{29}$

Four studies measured unprotected sex acts. $\begin{array}{lll}20 & 26 & 30 \\ \text { Two }\end{array}$ reported absolute reductions of $23.0 \% 0^{27}$ to increase of $0.8 \%$. $^{20}$ A third reported a $24.2 \%$ relative decrease in the number of unprotected sex acts $^{30}$ after testing. The condom use was assessed through a 'sexual behaviour score', ${ }^{26}$ approximating the likelihood of HIV acquisition using the frequencies of 
Table 1 Characteristics of studies included for qualitative synthesis

\begin{tabular}{|c|c|c|c|c|c|c|c|c|c|}
\hline \multirow[b]{2}{*}{ Study } & \multicolumn{3}{|l|}{ Data } & \multirow{2}{*}{$\begin{array}{l}\text { Was } \\
\text { behaviour } \\
\text { primary } \\
\text { purpose? }\end{array}$} & \multirow[b]{2}{*}{$\begin{array}{l}\text { Study } \\
\text { design }\end{array}$} & \multirow[b]{2}{*}{ Geographic setting } & \multirow[b]{2}{*}{$\begin{array}{l}\text { Study population } \\
\text { characteristics }\end{array}$} & \multirow[b]{2}{*}{$\begin{array}{l}\text { Age range } \\
\text { and gender }\end{array}$} & \multirow[b]{2}{*}{$\begin{array}{l}\text { Potential } \\
\text { biases* }\end{array}$} \\
\hline & $\begin{array}{l}\text { Condom } \\
\text { use }\end{array}$ & $\begin{array}{l}\text { No. of } \\
\text { partners }\end{array}$ & $\begin{array}{l}\text { Sex } \\
\text { acts }\end{array}$ & & & & & & \\
\hline Gray et $a l^{19}$ & Y & $Y$ & & No & $\mathrm{RCT}$ & Rakai, Uganda (R) & General population & 15-49, male & $\begin{array}{l}\text { SD, } \\
\text { sampling, } \\
\text { recall }\end{array}$ \\
\hline Kiene et $a l^{20}$ & Y & $\mathrm{N}$ & & Yes & $\begin{array}{l}\text { Prospective } \\
\text { cohort }\end{array}$ & $\begin{array}{l}\text { Mpigi District, } \\
\text { Uganda (R) }\end{array}$ & Outpatients & $\begin{array}{l}18+\text {, male and } \\
\text { female }\end{array}$ & $\begin{array}{l}\text { Sampling, } \\
\text { recall }\end{array}$ \\
\hline Ruzagira et $a l^{21}$ & Y & $Y$ & & No & $\begin{array}{l}\text { Prospective } \\
\text { cohort }\end{array}$ & Masaka, Uganda (R) & $\begin{array}{l}\text { HIV partners in } \\
\text { serodiscordant } \\
\text { relationships }\end{array}$ & $\begin{array}{l}\text { 18-60, male } \\
\text { and female }\end{array}$ & $\begin{array}{l}\text { SD, } \\
\text { sampling, } \\
\text { recall }\end{array}$ \\
\hline Wawer et $a l^{22}$ & Y & Y & & No & $\mathrm{RCT}$ & Rakai, Uganda (R) & General population & 15-49, female & $\begin{array}{l}\text { SD, } \\
\text { sampling, } \\
\text { recall }\end{array}$ \\
\hline Ndase et $a l^{23}$ & $\mathrm{~N}$ & Y & & Yes & $\begin{array}{l}\text { Prospective } \\
\text { cohort }\end{array}$ & $\begin{array}{l}\text { Botswana, Kenya, } \\
\text { Rwanda, SA, Tanzania, } \\
\text { Uganda and Zambia } \\
\text { (14 sites) }\end{array}$ & $\begin{array}{l}\text { HIV partners in } \\
\text { serodiscordant } \\
\text { relationships }\end{array}$ & $\begin{array}{l}18+\text {, male and } \\
\text { female }\end{array}$ & $\begin{array}{l}\text { SD, } \\
\text { sampling }\end{array}$ \\
\hline Bechange et $a l^{24}$ & $Y$ & $\mathrm{~N}$ & & Yes & $\begin{array}{l}\text { Prospective } \\
\text { cohort }\end{array}$ & $\begin{array}{l}\text { Tororo and Busia } \\
\text { districts, Uganda (R) }\end{array}$ & $\begin{array}{l}\text { Non-spousal } \\
\text { household members } \\
\text { of those commencing } \\
\text { ART† }\end{array}$ & $\begin{array}{l}\text { 18-69, male } \\
\text { and female }\end{array}$ & $\begin{array}{l}\text { SD, } \\
\text { sampling, } \\
\text { recall, } \\
\text { attrition }\end{array}$ \\
\hline Mugwanya et al ${ }^{25}$ & $Y$ & $\mathrm{~N}$ & & Yes & $\mathrm{RCT}$ & $\begin{array}{l}\text { Kenya: Eldoret, } \\
\text { Kisumu, Nairobi and } \\
\text { Thika (U) } \\
\text { Uganda: Jinja, } \\
\text { Kabwohe, Kampala, } \\
\text { Mbale, Tororo (U) }\end{array}$ & $\begin{array}{l}\text { HIV partners in } \\
\text { serodiscordant } \\
\text { relationships }\end{array}$ & $\begin{array}{l}\text { 18-65, male } \\
\text { and female }\end{array}$ & $\begin{array}{l}\text { SD, } \\
\text { sampling }\end{array}$ \\
\hline Ritchie et $a l^{26}$ & Y & $\mathrm{N}$ & & Yes & $\begin{array}{l}\text { Prospective } \\
\text { cohort }\end{array}$ & Entebbe, Uganda (U) & $\begin{array}{l}\text { HIV partners in } \\
\text { serodiscordant and } \\
\text { seroconcordant } \\
\text { relationships }\end{array}$ & $\begin{array}{l}18+\text {, male and } \\
\text { female }\end{array}$ & $\begin{array}{l}\text { SD, } \\
\text { sampling, } \\
\text { recall }\end{array}$ \\
\hline Kalichman et $a l^{27}$ & Y & Y & & Yes & $\begin{array}{l}\text { Prospective } \\
\text { cohort }\end{array}$ & Cape Town, SA (U) & STI clinic attendees & $\begin{array}{l}\text { Unspecified, } \\
\text { male and } \\
\text { female }\end{array}$ & Sampling \\
\hline Ramjee et $a l^{28}$ & Y & $\mathrm{Y}$ & & No & $\begin{array}{l}\text { Prospective } \\
\text { cohort }\end{array}$ & $\begin{array}{l}\text { Durban, SA (U); } \\
\text { Hlabisa, SA (R); } \\
\text { Lusaka, Zambia (U); } \\
\text { Moshi, Tanzania }(U+R)\end{array}$ & $\begin{array}{l}\text { Sexually active } \\
\text { women, largely from } \\
\text { general population }\end{array}$ & $\begin{array}{l}\text { Tanzania and } \\
\text { Zambia: } 16+, \\
\text { female } \\
\text { SA: } 18+\text {, } \\
\text { female }\end{array}$ & $\begin{array}{l}\text { SD, } \\
\text { sampling }\end{array}$ \\
\hline Padian et $a l^{29}$ & $Y$ & $\mathrm{~N}$ & & No & $\mathrm{RCT}$ & $\begin{array}{l}\text { Durban, SA (U); } \\
\text { Johannesburg, SA (U); } \\
\text { Harare, Zimbabwe (U) }\end{array}$ & $\begin{array}{l}\text { Sexually active } \\
\text { women from general } \\
\text { population }\end{array}$ & $18-49$, female & $\begin{array}{l}\text { SD, } \\
\text { sampling, } \\
\text { recall }\end{array}$ \\
\hline Van Damme et $a l^{30}$ & $Y$ & Y & & No & $\mathrm{RCT}$ & $\begin{array}{l}\text { Bondo, Kenya }(U) ; \\
\text { Pretoria, SA (U); } \\
\text { Bloemfontein, SA (U); } \\
\text { Arusha, Tanzania }(U)\end{array}$ & $\begin{array}{l}\text { High risk } \ddagger \text { members } \\
\text { from general } \\
\text { population }\end{array}$ & 18-35, female & $\begin{array}{l}\text { SD, } \\
\text { sampling }\end{array}$ \\
\hline Djomand et $a l^{31}$ & Y & Y & & Yes & $\begin{array}{l}\text { Prospective } \\
\text { cohort }\end{array}$ & $\begin{array}{l}\text { Gaborone, Botswana } \\
\text { (U) }\end{array}$ & $\begin{array}{l}\text { High risk§ } \\
\text { heterosexual men } \\
\text { and women }\end{array}$ & $\begin{array}{l}\text { 18-35, male } \\
\text { and female }\end{array}$ & $\begin{array}{l}\text { SD, } \\
\text { sampling, } \\
\text { recall }\end{array}$ \\
\hline Matambo et $a l^{32}$ & $Y$ & $\mathrm{~N}$ & & Yes & $\begin{array}{l}\text { Prospective } \\
\text { cohort }\end{array}$ & Harare, Zimbabwe (U) & Employees & $\begin{array}{l}\text { Unspecified, } \\
\text { mostly male }\end{array}$ & $\begin{array}{l}\text { SD, } \\
\text { sampling, } \\
\text { recall }\end{array}$ \\
\hline
\end{tabular}

different types of unprotected sex acts. It found that HIV-negative persons in serodiscordant and seroconcordant relationships underwent relative decreases in their scores of $100 \%$ and 25\%, respectively (representing increased condom use and/or reduced sexual activity).

With respect to population-specific findings, three studies assessed the general population recruited from the community. Two found negligible changes $(\leq 1 \%)$ in consistent or zero condom use after testing, ${ }^{22} 28$ while the third saw an overall decrease of $3.0 \%$ in consistent condom use ${ }^{19}$ post-testing. Two studies examined condom use in serodiscordant partnerships. One found a reduction in consistent condom use of $10.6 \%$ but $40.0 \%$ more people reported using condoms at least once (ie, reduction in zero condom use), ${ }^{21}$ while the second, a randomised controlled trial examining behaviour change post-testing in the context of HIV pre-exposure prophylaxis, found an $18 \%$ 
Table 2 Summary of results for studies assessing condom use before and after testing for HIV

\begin{tabular}{|c|c|c|c|c|c|c|}
\hline Study & Condom outcome measure & Sample size & $\begin{array}{l}\text { Baseline value } \\
\text { of outcome }\end{array}$ & $\begin{array}{l}\text { Absolute change } \\
\text { after testing }\end{array}$ & $\begin{array}{l}\text { Follow-up time } \\
\text { (months) }\end{array}$ & $\mathrm{p}$ Value \\
\hline \multicolumn{7}{|l|}{ Consistent condom use } \\
\hline Gray et $a l^{19}$ & Consistent use in last year & $\begin{array}{l}2474 \text { (circumcised) } \\
2522 \text { (uncircumcised) }\end{array}$ & $\begin{array}{l}32.6 \% \\
33.7 \%\end{array}$ & $\begin{array}{l}3.1 \% \text { decrease } \\
3.0 \% \text { decrease }\end{array}$ & 24 & NR \\
\hline Ruzagira et $a l^{21}$ & Always used in last year & 495 & $16.4 \%$ & $10.6 \%$ decrease & 24 & NR \\
\hline Wawer et $a l^{22}$ & Consistent use in last year & $\begin{array}{l}648 \text { (with circumcised partners) } \\
597 \text { (with uncircumcised } \\
\text { partners) }\end{array}$ & $\begin{array}{l}0.3 \% \\
0.8 \%\end{array}$ & $\begin{array}{l}0.8 \% \text { increase } \\
0.2 \% \text { decrease }\end{array}$ & 24 & NR \\
\hline Kiene et $a l^{20}$ & $\begin{array}{l}100 \% \text { use with risky partner* } \\
\text { in last } 3 \text { months }\end{array}$ & 131 (risky participants) & NR & $7.6 \%$ increase & 3 & NR \\
\hline Ramjee et $a l^{28}$ & $100 \%$ use in last week & 958 & $49.2 \%$ & $0.1 \%$ decrease & 12 & NR \\
\hline Matambo et $a l^{32}$ & $\begin{array}{l}\text { No unprotected sex in last } \\
3 \text { months }\end{array}$ & 388 & $17.5 \%$ & $0.2 \%$ decrease & 3 & 0.84 \\
\hline \multicolumn{7}{|l|}{ No condom use } \\
\hline Gray et $a l^{19}$ & Not used in last year & $\begin{array}{l}2474 \text { (circumcised) } \\
2522 \text { (uncircumcised) }\end{array}$ & $\begin{array}{l}39.5 \% \\
37.3 \%\end{array}$ & $\begin{array}{l}3.1 \% \text { decrease } \\
1.2 \% \text { decrease }\end{array}$ & 24 & NR \\
\hline Ruzagira et $a l^{21}$ & Not used in last year & 495 & $40.0 \%$ & $40.0 \%$ decrease & 24 & NR \\
\hline Wawer et $a l^{22}$ & Not used in last year & $\begin{array}{l}648 \text { (with circumcised partners) } \\
597 \text { (with uncircumcised } \\
\text { partners) }\end{array}$ & $\begin{array}{l}85.5 \% \\
81.2 \%\end{array}$ & $\begin{array}{l}1.0 \% \text { decrease } \\
2.8 \% \text { decrease }\end{array}$ & 24 & NR \\
\hline Ramjee et $a l^{28}$ & Not used in last week & 958 & $45.4 \%$ & $0.7 \%$ increase & 12 & NR \\
\hline Padian et $a l^{29}$ & $\begin{array}{l}\% \text { did not use condoms at any } \\
\text { time in last } 3 \text { months }\end{array}$ & $\begin{array}{l}2523 \text { (interventiont) } \\
2522 \text { (controlt) }\end{array}$ & $\begin{array}{l}30 \% \\
30 \%\end{array}$ & $\begin{array}{l}19 \% \text { decrease } \\
25 \% \text { decrease }\end{array}$ & 24 & $\begin{array}{l}<0.0001 \\
<0.0001\end{array}$ \\
\hline \multicolumn{7}{|l|}{ Unprotected sex } \\
\hline Bechange et $a l^{24}$ & $\begin{array}{l}\% \text { reporting risky sex in last } \\
3 \text { months }\end{array}$ & 236 (sexually active) & $30.9 \%$ & $16.6 \%$ decrease & 24 & NR \\
\hline Mugwanya et $a l^{25} \S$ & $\begin{array}{l}\% \text { reporting unprotected sex } \\
\text { with HIV+partner in last } \\
\text { month }\end{array}$ & $\begin{array}{l}4747 \text { (taking pre-exposure } \\
\text { prophylaxis) }\end{array}$ & $27.3 \%$ & $18 \%$ decrease & 24 & NR \\
\hline Djomand et $a l^{31}$ & $\begin{array}{l}\% \text { reporting unprotected sex in } \\
\text { last } 6 \text { months }\end{array}$ & $\begin{array}{l}64 \text { (males) } \\
33 \text { (females) }\end{array}$ & $\begin{array}{l}66.7 \% \\
60.6 \%\end{array}$ & $\begin{array}{l}10.0 \% \text { decrease } \\
17.1 \% \text { decrease }\end{array}$ & 12 & NR \\
\hline Padian et $a l^{29}$ & $\begin{array}{l}\% \text { did not use condom at last } \\
\text { sex }\end{array}$ & $\begin{array}{l}2523 \text { (interventiont) } \\
2522 \text { (controlt) }\end{array}$ & $\begin{array}{l}32 \% \\
33 \%\end{array}$ & $\begin{array}{l}8 \% \text { increase } \\
15 \% \text { decrease }\end{array}$ & 24 & $\begin{array}{l}\text { NR } \\
\text { NR }\end{array}$ \\
\hline \multicolumn{7}{|c|}{ Measures of unprotected acts } \\
\hline Kiene et $a l^{20}$ & $\begin{array}{l}\% \text { of risky acts* that were } \\
\text { unprotected in the last } \\
3 \text { months }\end{array}$ & 213 & $93.1 \%$ & $0.8 \%$ increase & & \\
\hline Kalichman et $a l^{27}$ & $\begin{array}{l}\% \text { of acts for which condoms } \\
\text { not used in last } 30 \text { days }\end{array}$ & $\begin{array}{l}29 \text { (seroconverted after initial } \\
\text {-ve test) } \\
77 \text { (persistently HIV-) }\end{array}$ & $\begin{array}{l}33.1 \% \\
33.3 \%\end{array}$ & $\begin{array}{l}24.0 \% \text { decrease } \\
22.6 \% \text { decrease }\end{array}$ & 12 & $\begin{array}{l}<0.01 \\
<0.01\end{array}$ \\
\hline Van Damme et $a l^{30}$ & $\begin{array}{l}\text { Number of sex acts without } \\
\text { condom in past week }\end{array}$ & 2120 & 1.9 acts & $\begin{array}{l}\text { Relative decrease by } \\
24.2 \%\end{array}$ & 12 & $<0.001$ \\
\hline Ritchie et $a l^{26}$ & $\begin{array}{l}\text { Median sexual behaviour } \\
\text { scoreq }\end{array}$ & $\begin{array}{l}72 \text { (serodiscordant) } \\
28 \text { (seroconcordant) }\end{array}$ & $\begin{array}{l}0.0085 \\
0.024\end{array}$ & $\begin{array}{l}\text { Relative decrease of } \\
100.0 \% \\
\text { Relative decrease of } \\
25.0 \%\end{array}$ & 9 & NR \\
\hline
\end{tabular}

Baseline percentages are calculated as the percentage of people reporting a given outcome. Changes after testing represent absolute changes unless explicitly reported as relative.

${ }^{*}$ Kiene et $a{ }^{20}$ risky sex is sex with a partner who is HIV-positive or of unknown serostatus.

tIn Padian et a ${ }^{29}$ intervention participants received a diaphragm, lubricant and male condoms, while control arm participants received only male condoms.

$\ddagger$ Bechange et $a^{24}$ defined risky sex as "risky sex as intercourse with inconsistent condom use with an HIV-infected partner or a partner of unknown serostatus during the prior 3 months".

$\S$ Additional data obtained from Baeten et al. ${ }^{39}$

IRitchie et al define a sexual behaviour score by the number of different types of unprotected sex acts over the last 3 months weighted by the likelihood of HIV acquisition by the given

type of sex act. Note that, unlike in Gray et $a l^{19}$ and Ramjee et $a l^{28}$ we include individuals reporting no sexual activity as having $100 \%$ condom use.

NR, not reported.

decrease in reported unprotected sex. ${ }^{25}$ Among individuals in serodiscordant partnerships, ${ }^{26}$ the 'sexual behaviour score' decreased to zero, meaning no unprotected sex acts were reported post-testing.

With the exception of the two studies among serodiscordant couples, overall there was no pattern observed between the direction of condom use and study factors such as sample size, risk of bias, study population characteristics, purpose of study, provision of free condoms during HIV testing and the nature or number of risk reduction counselling sessions involved. This was assessed through counting the proportion of positive outcomes. In all studies reporting multiple follow-ups, post-test condom use appeared stable over different lengths of follow-up 192224252931 (see online supplementary material).

\section{HIV testing and number of sex partners}

The effects of HIV testing on the number of sex partners were variable $(n=8$, table 3 ; additional time-points in online 
Table 3 Summary of results for studies assessing number of partners before and after testing for HIV

\begin{tabular}{|c|c|c|c|c|c|c|}
\hline Study & Outcome measure & Sample size & $\begin{array}{l}\text { Baseline value } \\
\text { of outcome }\end{array}$ & $\begin{array}{l}\text { Change after } \\
\text { testing }\end{array}$ & $\begin{array}{l}\text { Follow-up time } \\
\text { (months) }\end{array}$ & p Value \\
\hline \multicolumn{7}{|c|}{ Multiple partnerships and concurrency } \\
\hline Gray et $a l^{19}$ & $2+$ partners in last year & $\begin{array}{l}2474 \text { (circumcised) } \\
2522 \text { (uncircumcised) }\end{array}$ & $\begin{array}{l}34.5 \% \\
34.1 \%\end{array}$ & $\begin{array}{l}1.0 \% \text { increase } \\
1.3 \% \text { increase }\end{array}$ & 24 & NR \\
\hline Wawer et $a l^{22}$ & $2+$ partners in last year & $\begin{array}{l}648 \text { (with circumcised } \\
\text { partners) } \\
597 \text { (with uncircumcised } \\
\text { partners) }\end{array}$ & $\begin{array}{l}3.5 \% \\
3.7 \%\end{array}$ & $\begin{array}{l}0.1 \% \text { decrease } \\
0.9 \% \text { increase }\end{array}$ & 24 & NR \\
\hline Ramjee et $a l^{28}$ & $2+$ partners in last 3 months & 958 & $1.7 \%$ & $1.0 \%$ decrease & 12 & NR \\
\hline Djomand et $a l^{31}$ & $\begin{array}{l}2+\text { partners in last } 6 \text { months } \\
4+\text { partners in last } 6 \text { months }\end{array}$ & $\begin{array}{l}33 \text { (females) } \\
64 \text { (males) }\end{array}$ & $\begin{array}{l}66.7 \% \\
21.9 \%\end{array}$ & $\begin{array}{l}58.0 \% \text { decrease } \\
16.9 \% \text { decrease }\end{array}$ & 12 & NR \\
\hline Ndase et $a l^{23}$ & $\begin{array}{l}\text { Concurrency in last month } \\
\text { Sex with outside partner in last } \\
\text { month }\end{array}$ & $\begin{array}{l}2284 \text { (males) } \\
1097 \text { (females) } \\
2284 \text { (males) } \\
1097 \text { (females) }\end{array}$ & $\begin{array}{l}4.1 \% \\
0.5 \% \\
4.3 \% \\
0.5 \%\end{array}$ & $\begin{array}{l}2.6 \% \text { increase } \\
\text { No change } \\
14.8 \% \text { increase } \\
3.5 \% \text { increase }\end{array}$ & 24 & $\begin{array}{l}\text { NR } \\
\text { NR } \\
<0.001 \\
<0.001\end{array}$ \\
\hline $\begin{array}{l}\text { Ruzagira et } a^{21} \\
\text { Monogamy or no pa }\end{array}$ & $\begin{array}{l}\text { Extramarital sex in last year } \\
\text { ners }\end{array}$ & 495 & $35.7 \%$ & $8.3 \%$ increase & 24 & NR \\
\hline Ndase et $a l^{23}$ & Sex with HIV+ primary partner only & $\begin{array}{l}2284 \text { (males) } \\
1097 \text { (females) }\end{array}$ & $\begin{array}{l}90.1 \% \\
91.6 \%\end{array}$ & $\begin{array}{l}23.8 \% \text { decrease } \\
18.1 \% \text { decrease }\end{array}$ & 24 & $\begin{array}{l}\text { NR } \\
\text { NR }\end{array}$ \\
\hline Gray et al ${ }^{19}$ & No partners in last year & $\begin{array}{l}2474 \text { (circumcised) } \\
2522 \text { (uncircumcised) }\end{array}$ & $\begin{array}{l}18.9 \% \\
19.6 \%\end{array}$ & $\begin{array}{l}5.5 \% \text { decrease } \\
5.0 \% \text { decrease }\end{array}$ & 24 & NR \\
\hline Wawer et $a l^{22}$ & No partners in last year & $\begin{array}{l}648 \text { (with circumcised } \\
\text { partners) } \\
597 \text { (with uncircumcised } \\
\text { partners) }\end{array}$ & $\begin{array}{l}0.0 \% \\
0.3 \%\end{array}$ & $\begin{array}{l}0.5 \% \text { increase } \\
0.1 \% \text { decrease }\end{array}$ & 24 & NR \\
\hline $\begin{array}{l}\text { Ramjee et } a l^{28} \\
\text { Number of partners }\end{array}$ & No partners in last 3 months & 958 & $0.0 \%$ & $5.9 \%$ increase & 12 & $<0.01$ \\
\hline Kalichman et $a l^{27}$ & $\begin{array}{l}\text { Mean number of partners in last } \\
30 \text { days }\end{array}$ & $\begin{array}{l}29 \text { (seroconverters) } \\
77 \text { (persistently HIV-negative) }\end{array}$ & $\begin{array}{l}2.2 \\
1.7\end{array}$ & $\begin{array}{l}\text { Relative decrease by } \\
59.1 \% \\
\text { Relative decrease by } \\
23.5 \%\end{array}$ & 12 & $<0.01$ \\
\hline Van Damme et al ${ }^{30}$ & Number of partners in past week & 2120 & 1.0 & $\begin{array}{l}\text { Relative decrease of } \\
14 \%\end{array}$ & 12 & $<0.001$ \\
\hline
\end{tabular}

supplement table S2). Four of eight studies noted decreases in partner numbers, and two reported more partners after HIV testing. One found changes of $<1 \%$ for all outcomes. ${ }^{22}$ As with studies of condom use, there was no discernible pattern underlying the variability.

In the two studies reporting mean number of sexual partners, both found statistically significant relative decreases of $35.2 \%{ }^{27}$ and $14.0 \% .^{30}$ Four studies assessed changes in the proportion of participants with more than one current partner. Three studies found a decrease of $1.0 \%$ to an increase of $1.1 \%,{ }^{19} 2228$ but two of them had few participants reporting multiple partners at baseline $\left(3.6 \%{ }^{22}\right.$ and $\left.1.7 \%{ }^{28}\right)$. The fourth recorded a large decrease of $58.0 \%$ in the proportion of women reporting two or more partners a year after testing. ${ }^{31}$

Three studies measured the proportion of participants reporting no partners, ${ }^{1922}{ }^{28}$ finding an increase of $5.9 \% 0^{28}$ to a decrease of $5.3 \%{ }^{19}$ post-testing, while the third found all changes $<1 \%$. $^{22}$

Studies of the general population recruited from the community also demonstrated variability. One study found negligible changes in the number of partners after testing (all changes $<1 \%$, p value not reported). ${ }^{22}$ Another found a statistically significant trend towards individuals reporting no partners $(5.9 \%$ increase, $\mathrm{p}<0.01) .{ }^{28}$ The third study found changes in number of partners $(5.3 \%$ decrease in those reporting no partners and $1.1 \%$ increase in those reporting 2 or more partners post-testing). ${ }^{19}$
Four studies examined sexual partnerships outside the primary relationship. ${ }^{19}$ 21-23 Two found that $16.1 \%{ }^{19}$ fewer to $1.2 \%{ }^{22}$ more participants reported extramarital sexual partnerships post-testing compared with baseline. The remaining two studies examined serodiscordant primary relationships specifically. $^{21}$ and found an increase of $8.3 \%$ in extramarital relationships post-testing. ${ }^{23}$ noted significant increases in those reporting sexual partners outside their primary relationship in the last month, from $3.1 \%$ at baseline to $13.9 \%$ after 24 months follow-up $(\mathrm{p}<0.001)$, with this increase mirrored by a steady decline throughout the quarterly follow-ups in the proportion of those reporting sex with their HIV-positive primary partner in the last month, possibly suggesting partner switching. ${ }^{23}$

\section{HIV testing and number of sex acts}

Five studies reported outcomes related to the number of sex acts (table 4; additional time-points in online supplementary table S3)..$^{20} 23252830$ Three studies reported on changes in individuals reporting no sex acts: two reported increases in individuals reporting no sex acts from $7.6 \%$ to $10.9 \%,{ }^{20}{ }^{23}$ while a third study reported a $7.1 \%$ reduction in individuals reporting sexual activity in the past week. ${ }^{28}$ Three studies reported on the post-testing change in the mean number of sex acts, ${ }^{20} 2530$ which ranged from a relative decrease of $15.7 \% 0^{30}$ to a relative increase of $9.4 \%{ }^{20}$ (increase in $2 / 3$ studies). 


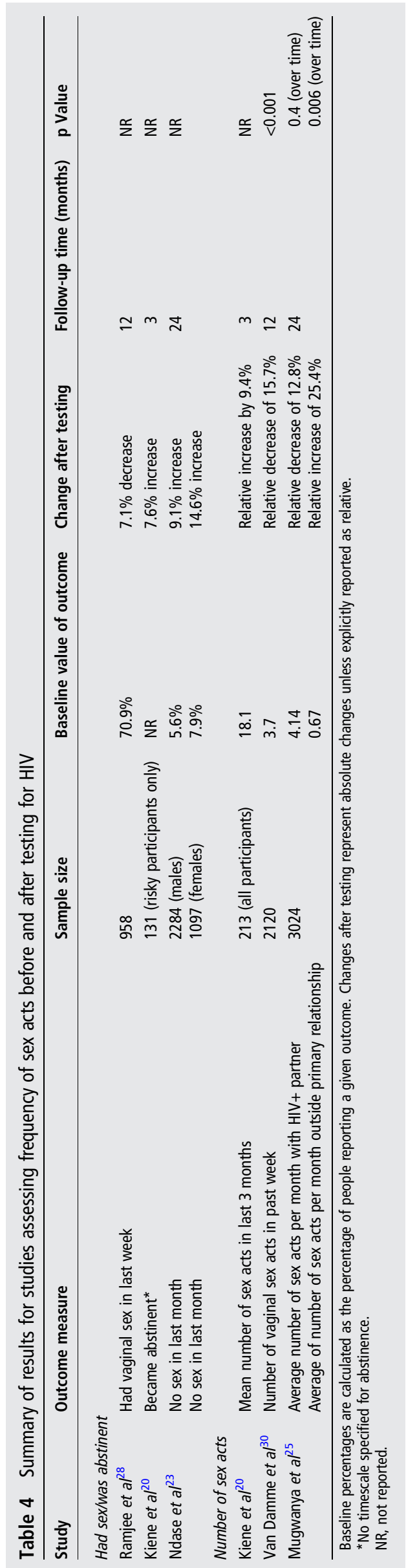

Of the above studies, one examined the general population recruited from the community, and found reduced vaginal intercourse $\left(7.1 \%\right.$ decrease, $\mathrm{p}$ value not reported). ${ }^{28}$ One study looked at changes in serodiscordant partnerships, measuring the average frequency of sex acts before and after trial participants found out if they were assigned to oral pre-exposure prophylaxis or placebo in the Partners PrEP study. It found that after unblinding the frequency of sex acts with the main HIV-positive partner decreased non-significantly, accompanied by a $25.4 \%$ relative increase in the frequency of sexual acts with another partner. ${ }^{25}$ Using a segmented regression model this increase was not significant immediately post-unblinding, but the change over time was significant $(p=0.006)$.

\section{DISCUSSION}

Fourteen primary studies carried out in SSA measured various sexual risk behaviour outcomes before and after HIV testing. Findings suggest that awareness of one's serostatus does not increase consistent condom use, but there were increases in the proportion of acts protected by a condom. Furthermore, in 3/4 studies there were increases in the proportion ever using a condom, suggesting that awareness of one's serostatus can shift HIV-negative individuals who never use condoms to start using them, although inconsistently. Condom use in serodiscordant partnerships generally increased: $2 / 3$ studies reported reduced risky sex, while in the third there was an increase in the proportion ever using a condom, although consistent condom use declined. While overall there was variability in the effects of HIV testing on the number of sex partners or sex acts among HIV-negative individuals, there was an increase in individuals reporting no sex acts in $3 / 3$ studies, and 2/3 studies reported increases in individuals reporting no sexual partners. Two studies saw significant decreases in the mean number of reported sexual partners. While a few studies reported large changes, the effect sizes observed were generally moderate. There was also little evidence of consistently increased risk behaviour following a negative HIV test, which may thus be reinforcing past behaviours and not increasing risk. In general, we could not identify any factors predicting behaviour modification. However, a notable exception was seen in HIV-negative partners in serodiscordant relationships, who appear to engage in more extrarelational sex after testing and counselling in studies examining this population, ${ }^{21} 2325$ although they may reduce condomless sex with their positive partner.

Our results complement those from the two systematic reviews and meta-analyses of HIV-negative individuals in low and middle-income countries (LMICs), ${ }^{11} 12$ although they did not examine serodiscordant couples. However, our review is more relevant to the present situation in SSA, since only 3 out of 17 studies were included, ${ }^{12}$ and no studies ${ }^{11}$ began enrolment after 2002.

Our findings, combined with results from other LMICs, ${ }^{11} 12$ may allow mathematical modellers to assume, with reasonable confidence, that risk behaviour among the HIV-negative majority does not markedly change post-testing. However, incorporating a sensitivity analysis to changes in risk behaviour is advisable, given the variability in our findings. Furthermore, findings among serodiscordant couples suggest that HIV testing may lead to changes in the sexual network due to partner switching by the seronegative partner. ${ }^{23}$

The results also have implications for universal testing and treatment. Across three studies assessing the general population recruited from the community, results suggest that although VCT does little to improve risk behaviour among individuals 
testing HIV-negative in that population, it importantly also does not increase risk behaviour. However, the lack of consistent evidence in either direction points to the need to incorporate suitable indicators in HIV surveillance to detect local increases in risk behaviour of HIV-negative individuals as HIV testing efforts intensify, so that additional risk reduction interventions may be put in place where necessary. In addition, few studies examined the effectiveness of different testing strategies. For instance, home-based counselling and testing has been shown to be highly acceptable in SSA, ${ }^{33}$ but our review failed to identify any studies evaluating its effectiveness in altering HIV-negative individuals' risk behaviour. Therefore, it is important to investigate the impact of new testing modalities on behaviour. More information is also needed on the impact of repeated testing, particularly in the long term, when individuals who have received multiple negative tests may perceive themselves to be at low risk of HIV acquisition.

Identification of serodiscordant couples is a high priority for global public health, as it allows provision of HIV prevention services including counselling about condoms and ART provision. ${ }^{34}$ However, this study highlights the need for more understanding of how the HIV risk behaviour of the seronegative partner may change. In particular, ART initiation and viral suppression of the HIV-positive partner may not mitigate HIV risk for the seronegative partner, who may be exposed through other partnerships after a negative HIV test result.

\section{Limitations}

There are limitations to this review. First, grey literature and programme data were not included. Second, other important behaviour changes associated with HIV testing, such as propensity to repeat test in the future, were not included. Third, only one study was a randomised controlled trial examining posttesting behaviour change; most data were subject to high risk of sampling and social desirability bias as they came from studies where post-testing behaviour was not the primary aim. Fourth, the quality and type of HIV counselling received when testing may differ. ${ }^{12} 35{ }^{36}$ Fifth, no study in this review measured pre-test intention. As individuals may resolve to reduce risky behaviour as part of the decision-making process to get tested, ${ }^{37}$ the behaviour change may differ if study recruitment

\section{Key messages}

- Further primary evidence with the primary purpose of examining behavioural change in HIV-negative individuals after testing is needed in sub-Saharan Africa (SSA), including in key populations.

- Additional research is needed to specifically investigate the behaviour of seronegative individuals in serodiscordant partnerships.

- As was previously found, ${ }^{12}$ there remains a need to standardise sexual behavioural outcomes to facilitate valid comparisons across studies and programmes.

- Future studies should aim to use methodologies that minimise sampling and social desirability biases. More qualitative evidence exploring the psychological determinants of behavioural change, or lack thereof, among HIV-negative individuals may help elucidate causal mechanisms underlying our observations. removes the active decision to test. Finally, both the study populations and the outcome measures varied widely, precluding our ability to pool data, and highlighting the need to more consistently measure pre-test and post-test behaviour.

\section{CONCLUSIONS}

Awareness of one's serostatus generally has a small effect on risk behaviour of HIV-negative individuals, although seronegative partners in serodiscordant partnerships may engage in more extrarelational sex. Additional research specifically examining behavioural change is needed in SSA, including in key populations and serodiscordant partnerships.

Acknowledgements We would like to thank the following who responded to our inquiries with additional study information and study clarifications: Adam Ritchie (University of Oxford) and the CHAVI 002 study team; Ashley Clayton (Fred Hutchinson Cancer Research Center), the HIV Vaccine Trials Network and the HVTN 903 study team. MP thanks the National Institutes of Health for funding through the NIAID cooperative agreement UM1AI068619. SM is supported by a Canadian Institutes of Health Research and Ontario HIV Treatment Network New Investigator Award. We thank Jacqueline Cousins, Imperial College Library Information Specialist, in supporting the generation of search terms.

Contributors MP and SM designed the study. SR and NC carried out the screening, full-text review and data extraction. SR, SM and MP undertook the analysis and interpretation of results. SR, SM and MP wrote the first draft of the report. All authors contributed to subsequent drafts of the report and reviewed the final version before submission.

Competing interests None declared.

Provenance and peer review Not commissioned; externally peer reviewed.

Open Access This is an Open Access article distributed in accordance with the Creative Commons Attribution Non Commercial (CC BY-NC 4.0) license, which permits others to distribute, remix, adapt, build upon this work non-commercially, and license their derivative works on different terms, provided the original work is properly cited and the use is non-commercial. See: http://creativecommons.org/ licenses/by-nc/4.0/

\section{REFERENCES}

1 World Health Organization. Data on the size of the HIV/AIDS epidemic: Data by WHO region. Secondary Data on the size of the HIVIAIDS epidemic: Data by WHO region. http://apps.who.int/gho/data/node.main.619?lang=en

2 Cohen MS, Chen YQ, McCauley M, et al. Prevention of HIV-1 infection with early antiretroviral therapy. N Engl J Med 2011;365:493-505.

3 UNAIDS. 90-90-90-An ambitious treatment target to help end the AIDS epidemic. Geneva: UNAIDS, 2014.

4 Auerbach JD. Principles of positive prevention. J Acquir Immune Defic Syndr 2004;37(Suppl 2):S122-5.

5 Granich RM, Gilks CF, Dye C, et al. Universal voluntary HIV testing with immediate antiretroviral therapy as a strategy for elimination of HIV transmission: a mathematical model. Lancet 2009;373:48-57.

6 Dodd PJ, Garnett GP, Hallett TB. Examining the promise of HIV elimination by 'test and treat' in hyperendemic settings. AIDS 2010;24:729-35.

7 Baggaley RF, Fraser C. Modelling sexual transmission of HIV: testing the assumptions, validating the predictions. Curr Opin HIV AIDS 2010;5:269-76.

8 Hallett TB, Dube $S$, Cremin I, et al. The role of testing and counselling for HIV prevention and care in the era of scaling-up antiretroviral therapy. Epidemics 2009;1:77-82.

9 Cori A, Ayles H, Beyers N, et al. HPTN 071 (PopART): a cluster-randomized trial of the population impact of an HIV combination prevention intervention including universal testing and treatment: mathematical model. PLOS ONE 2014;9:e84511.

10 Denison JA, O'Reilly KR, Schmid GP, et al. HIV voluntary counseling and testing and behavioral risk reduction in developing countries: a meta-analysis, 1990-2005. AIDS Behav 2008;12:363-73.

11 Kennedy CE, Medley AM, Sweat MD, et al. Behavioural interventions for HIV positive prevention in developing countries: a systematic review and meta-analysis. Bull World Health Organ 2010;88:615-23.

12 Fonner VA, Denison J, Kennedy CE, et al. Voluntary counseling and testing (VCT) for changing HIV-related risk behavior in developing countries. Cochrane Database Syst Rev 2012;9:CD001224.

13 World Health Organization, UNAIDS, UNICEF. Towards universal access: scaling up priority HIVIAIDS interventions in the health sector: progress report, April 2007. Geneva: WHO, 2007.

14 Moher D, Liberati A, Tetzlaff J, et al. Preferred reporting items for systematic reviews and meta-analyses: the PRISMA statement. PLoS Med 2009;6:e1000097. 
15 United Nations Statistics Division. Composition of macro geographical (continental) regions, geographical sub-regions, and selected economic and other groupings. Secondary Composition of macro geographical (continental) regions, geographical sub-regions, and selected economic and other groupings. https://unstats.un.org/ unsd/methods/m49/m49regin.htm

16 Napper LE, Fisher DG, Reynolds GL, et al. HIV risk behavior self-report reliability at different recall periods. AIDS Behav 2010;14:152-61.

17 Schroder KE, Carey MP, Vanable PA. Methodological challenges in research on sexual risk behavior: II. Accuracy of self-reports. Ann Behav Med 2003;26:104-23.

18 Higgins JPT, Green S, Cochrane Collaboration. Cochrane handbook for systematic reviews of interventions. Chichester, England; Hoboken, NJ: Wiley-Blackwell, 2008.

19 Gray RH, Kigozi G, Serwadda D, et al. Male circumcision for HIV prevention in men in Rakai, Uganda: a randomised trial. Lancet 2007;369:657-66.

20 Kiene SM, Bateganya M, Wanyenze R, et al. Initial outcomes of provider-initiated routine HIV testing and counseling during outpatient care at a rural Ugandan hospital: risky sexual behavior, partner HIV testing, disclosure, and HIV care seeking. AIDS Patient Care STDS 2010;24:117-26.

21 Ruzagira $E$, Wandiembe $S$, Abaasa A, et al. HIV incidence and risk factors for acquisition in HIV discordant couples in Masaka, Uganda: an HIV vaccine preparedness study. PLOS ONE 2011;6:e24037.

22 Wawer MJ, Tobian AA, Kigozi G, et al. Effect of circumcision of HIV-negative men on transmission of human papillomavirus to HIV-negative women: a randomised trial in Rakai, Uganda. Lancet 2011;377:209-18.

23 Ndase P, Celum C, Thomas K, et al. Outside sexual partnerships and risk of HIV acquisition for HIV uninfected partners in African HIV serodiscordant partnerships. J Acquir Immune Defic Syndr 2012;59:65-71.

24 Bechange S, Bunnell R, Awor A, et al. Two-year follow-up of sexual behavior among HIV-uninfected household members of adults taking antiretroviral therapy in Uganda: no evidence of disinhibition. AIDS Behav 2010;14:816-23.

25 Mugwanya KK, Donnell D, Celum C, et al. Sexual behaviour of heterosexual men and women receiving antiretroviral pre-exposure prophylaxis for HIV prevention: a longitudinal analysis. Lancet Infect Dis 2013;13:1021-8.

26 Ritchie AJ, Kuldanek K, Moodie Z, et al. Comparison of sexual behavior and HIV risk between two HIV-1 serodiscordant couple cohorts: the CHAVI 002 study. PLOS ONE 2012;7:e37727.
27 Kalichman SC, Cain D, Simbayi LC. Behavioral changes associated with testing HIV-positive among sexually transmitted infection clinic patients in Cape Town, South Africa. Am J Public Health 2010;100:714-19.

28 Ramjee $G$, Kapiga $S$, Weiss $S$, et al. The value of site preparedness studies for future implementation of phase 2/IIb/III HIV prevention trials: experience from the HPTN 055 study. J Acquir Immune Defic Syndr 2008;47:93-100.

29 Padian NS, van der Straten A, Ramjee G, et al. Diaphragm and lubricant gel for prevention of HIV acquisition in southern African women: a randomised controlled trial. Lancet 2007;370:251-61.

30 Van Damme L, Corneli A, Ahmed K, et al. Preexposure prophylaxis for HIV infection among African women. N Engl J Med 2012;367:411-22.

31 Djomand $G$, Metch B, Zorrilla CD, et al. The HVTN protocol 903 vaccine preparedness study: lessons learned in preparation for HIV vaccine efficacy trials. J Acquir Immune Defic Syndr 2008;48:82-9.

32 Matambo R, Dauya E, Mutswanga J, et al. Voluntary counseling and testing by nurse counselors: what is the role of routine repeated testing after a negative result? Clin Infect Dis 2006;42:569-71.

33 Sabapathy K, Van den Bergh R, Fidler S, et al. Uptake of home-based voluntary HIV testing in sub-Saharan Africa: a systematic review and meta-analysis. PLoS Med 2012;9:e1001351

34 Curran K, Baeten JM, Coates TJ, et al. HIV-1 prevention for HIV-1 serodiscordant couples. Curr HIVIAIDS Rep 2012;9:160-70.

35 Holtgrave D, McGuire J. Impact of counseling in voluntary counseling and testing programs for persons at risk for or living with HIV infection. Clin Infect Dis 2007;45 (Suppl 4):S240-3.

36 Ngure K, Mugo N, Celum C, et al. A qualitative study of barriers to consistent condom use among HIV-1 serodiscordant couples in Kenya. AIDS care 2012;24:509-16.

37 Sikasote J, Grant L, Chinn DJ, et al. Voluntary counselling and testing for HIV in a Zambian mining community: serial interviews with people testing negative. Sex Transm Infect 2011;87:433-8.

38 Fiorillo SP, Landman KZ, Tribble AC, et al. Changes in HIV risk behavior and seroincidence among clients presenting for repeat HIV counseling and testing in Moshi, Tanzania. AIDS care 2012;24:1264-71.

39 Baeten JM, Donnell D, Ndase P, et al. Antiretroviral prophylaxis for HIV prevention in heterosexual men and women. N Engl J Med 2012;367:399-410. 